\title{
EFFICIENT DYADIC WAVELET TRANSFORMATION OF IMAGES USING INTERPOLATION FILTERS
}

\author{
Michael Unser \\ Biomedical Engineering and Instrumentation Program, NCRR, Bldg. 13, Room 3W13 \\ National Institutes of Health, Bethesda, MD 20892 USA
}

\begin{abstract}
The properties of a special class of overcomplete wavelet transforms specified in terms of an interpolation filter are investigated. The decomposition is obtained by filtering the signal with a sequence of increasingly selective lowpass filters with a dyadic scale progression. The wavelet coefficients are evaluated by simple subtraction of two consecutive lowpass components. The lowpass filter bank is implemented using a standard iterative multiscale algorithm. The impulse responses of the analysis filters are shown to be interpolated versions of each other. This structure is computationally very efficient; it requires a little more than $1 / 4$ as many operations as other comparable wavelet-based algorithms. The corresponding filter bank provides a perfect coverage of the frequency domain which results in a trivial reconstruction procedure by summation. Extensions for the sub-sampled case are also presented. The decompositions associated with spline interpolation filters are considered in more details and some image processing examples are presented.
\end{abstract}

\section{INTRODUCTION}

The equivalence between perfect reconstruction filter banks and the discrete wavelet transform is now well recognized [1, 2]. In essence, these techniques provide a compact subband (or hierarchical) decomposition of signals and are therefore ideally suited for applications such as image coding. Despite the obvious appeal of these non-redundant representations, it appears that the use of overcomplete decompositions such as the dyadic wavelet transform may be better suited for certain tasks in signal analysis and image processing [3]. Recent examples of applications include edge detection, noise reduction, as well as the representation of signals by their maxima in the wavelet domain.

The simplest way to obtain such signal decompositions is to use a tree-structured algorithm by which each branch is divided into its lowpass and highpass components. This process typically requires the implementation of two complementary lowpass and highpass filters (for a redundant representation, one uses the "à trous" algorithm in which the filters are progressively expanded by insertion of zeros in-between taps [4]). Note that in the special case of an ideal lowpass filter, the highpass component can, in principle, be obtained by simple subtraction. This principle is also used explicitly in the difference-of-gaussian representation, which is commonly used for edge detection [5].

In this paper, we will build upon this simple subtraction idea and investigate a general class of discrete dyadic wavelet transforms that takes full advantage of this property and therefore results in very efficient implementation. These representations provide a subband decomposition with a perfect coverage of the frequency domain. This property results in a simple reconstruction algorithm by summation of the individual components. In this sense, the present construction is related to the multiresolution representation of Saito and Beyklin that uses the autocorrelation function of a Daubechies wavelet [6].

\section{THE BASIC DECOMPOSITION}

\subsection{Definition}

The decomposition algorithm is based on the specification of a single filter $h$ that satisfies the interpolation property $h(2 k)=\delta_{k}$. In the $z$-transform domain, this condition is expressed as

$$
\frac{1}{2}(H(z)+H(-z))=1 .
$$

We also impose the lowpass constraint: $H(1)=2 \Rightarrow H(-1)=0$. The corresponding highpass filter $g$ is obtained by simple modulation

$$
G(z)=H(-z) \text {. }
$$

Based on these prototypes, we construct a sequence of complementary lowpass and bandpass filters using the following recursive definition

$$
\begin{aligned}
& H_{(i)}(z)=\frac{1}{2} H\left(z^{2^{-1}}\right) H_{(i-1)}(z), \\
& G_{(i)}(z)=\frac{1}{2} G\left(z^{2^{+-1}}\right) H_{(i-1)}(z),
\end{aligned}
$$

\section{V-149}

U.S. Government work not protected by U.S. Copyright 


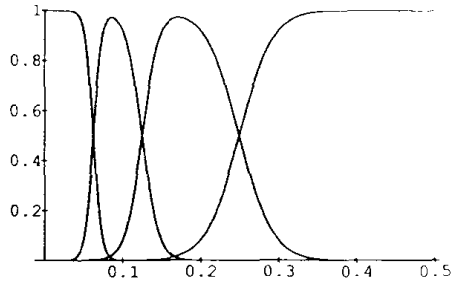

Fig. 1. Multiband characteristics of the filters associated with the quintic spline interpolator (c.f. Eq. (19) with $p=2$ ).

with the initial condition $H_{(0)}(z)=1$. The subscript (i) represents the scale index.

A discrete signal $\{s(k)\}_{k \in Z}$ is then characterized by its discrete dyadic wavelet transform $\left\{d_{(1)}, \cdots, d_{(l)}, s_{(l)}\right\}$, obtained by simple digital filtering (without decimation)

$$
\begin{aligned}
& s_{(i)}(k)=h_{(i)} * s(k) \\
& d_{(i)}(k)=g_{(i)} * s(k)
\end{aligned}
$$

where $h_{(i)}$ and $\mathrm{g}_{(i)}$ are the impulse responses of the filters defined by (3) and (4), respectively. The $d_{(i)}$ 's are the highpass wavelet coefficients (or details), while $s_{(I)}$ is the lowpass component at resolution ( $I$ (the depth of the decomposition).

\subsection{Properties}

By iterating (3), one finds that the transfer function of the lowpass filter at resolution $(i)$ is simply

$$
H_{\langle i\rangle}(z)=\frac{1}{2^{\prime}} \prod_{j=1}^{i} H\left(z^{2^{-1}}\right) .
$$

For the bandpass filter, we can use (4), (2) and (1) to derive the fundamental relation

$$
G_{(i)}(z)=H_{(i-1)}(z)-H_{(i)}(z),
$$

from which we deduce that

$$
H_{(i)}(z)+\sum_{j=1}^{i} G_{(j)}(z)=1 .
$$

This last equation essentially states that we have a full coverage of the frequency domain and that the representation can be viewed as a subband signal decomposition (c.f. Fig. 1).

The sequence of filters $h_{(i)}$ specified in Section 2.1 defines an interpolation filter bank. The use of this terminology is motivated by the following remarkable property

$$
\left[h_{(i)}\right]_{\downarrow 2^{j}}=2^{-j} h_{(i-j)} \text {, }
$$

where the operator []$_{\downarrow m}$ denotes the decimation by a factor of $m$. Eq. (10) indicates that the lowpass filters at finer scales can be obtained by decimation of filters corresponding to coarser resolutions (plus an appropriate normalization). Conversely, one can think off the sequence $h_{(i)}$ as an enlarged interpolated version of $h_{(i-j)}$, for $i>j>0$.

Proof: We start by down-sampling $h_{(i)}$ by a factor of two and express this operation in the z-transform domain

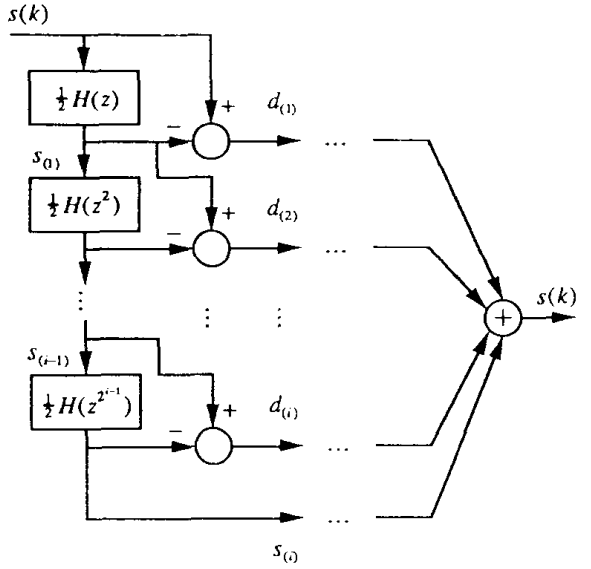

Fig. 2. Fast subband decomposition algorithm.

$$
\left[h_{(i)}\right]_{\downarrow 2}(k) \stackrel{z}{\longleftrightarrow} \tilde{H}_{(i)}(z)=\frac{1}{2}\left(H_{(i)}\left(z^{1 / 2}\right)+H_{(i)}\left(-z^{1 / 2}\right)\right) .
$$

Next, we replace $H_{(i)}(z)$ by its expression in (7) which gives

$$
\tilde{H}_{(i)}\left(z^{2}\right)=\frac{1}{z^{+1}} \prod_{j=2}^{i} H\left(z^{2^{\mu-1}}\right)(H(z)+H(-z)) .
$$

We then use the interpolation condition (1) and obtain

$$
\tilde{H}_{(i)}\left(z^{2}\right)=\frac{1}{z} \prod_{j=2}^{i} H\left(z^{2-1}\right)=\frac{1}{2} H_{(i-1)}\left(z^{2}\right),
$$

for which we conclude that

$$
\left[h_{(i)}\right]_{\downarrow 2}=\frac{1}{2} h_{(i-1)} \text {. }
$$

This decimation process is then repeated $(j-1)$ more times to get the desired result.

\subsection{Fast algorithms}

Using (3) and (8), it is straightforward to derive the fast iterative wavelet decomposition algorithm

$$
\left\{\begin{array}{l}
s_{(i)}(k)=\left[\frac{1}{2} h\right]_{\uparrow^{2}-1} * s_{(i-1)}(k) \\
d_{(i)}(k)=s_{(i-1)}(k)-s_{(i)}(k)
\end{array}\right.
$$

where the operator $[\cdot]_{\uparrow m}$ denotes the up-sampling by a factor of $m$. Note that the complexity of the algorithm, which is constant over all iterations, is essentially one fourth of that of the conventional approach (there is only one filter instead of two, and every other (even) filter coefficient is zero) The reconstruction algorithm is trivial and follows directly from (9)

$$
s(k)=s_{(i)}(k)+\sum_{j=1}^{i} d_{(j)}(k) .
$$

These properties result in a very efficient and simple implementation (c.f. Fig. 2).

\section{INTERPRETATIONS AND EXTENSIONS}

\subsection{The underlying interpolation kernel}

Instead of the discrete sequence $h_{(i)}(k)$, let us now consider the function of the continuous variable $x$, 


$$
\varphi_{(i)}(x)=2^{i} \cdot h_{(i)}(k), \quad k / 2^{i} \leq x<(k+1) / 2^{i},
$$

which is piecewise constant on intervals of length $1 / 2^{i}$. If the filter $h$ satisfies appropriate regularity conditions, then the sequence of functions $\varphi_{(i)}(x)$ converges to some continuous limit $\varphi(x)[7,8]$. This limit, if it exists, is a (non-orthogonal) scaling function in the sense that it satisfies the two-scale equation

$$
\varphi(x)=\sum_{k \in Z} h(k) \varphi(2 x-k) .
$$

Its Fourier transform can be obtained from the infinite product

$$
\hat{\varphi}(f)=\prod_{j=1}^{+\infty}\left(\frac{1}{2} H\left(e^{j 2 \times f 2^{-j}}\right)\right) .
$$

A necessary condition for this convergence to occur is that $H(z)$ has at least one zero at $z=-1$ (c.f. the lowpass constraint in Section 2.1). Because of the conditions initially imposed on $h$, the scaling function $\varphi(x)$ is an interpolation kernel; i.e. $\varphi(k)=\delta_{k}$. Moreover, the sequence of filters $h_{(i)}$ can be generated by sampling $\varphi(x)$ at the appropriate rate

$$
h_{i i}(k)=\frac{1}{2} \varphi\left(k / 2^{i}\right) \text {. }
$$

This result follows directly from the definition (13) and the discrete interpolation property (10). Finally, we note that the degree of smoothness (or regularity) of $\varphi(x)$ is controlled by the number of zeros of $H(z)$ at $z=-1[7,8]$.

An alternative procedure for constructing a symmetrical interpolation filterbank is to start with an orthogonal scaling $\phi(x)$ (in the sense defined in [9]) and construct the autocorrelation function $\varphi(x)=\phi(-x) * \phi(x)$. The fact that the resulting $\varphi(x)$ is a valid interpolation kernel is a direct consequence of the orthogonality of $\phi(x)$ with its integer translates. The corresponding discrete filter $h$ can be obtained by sampling $\varphi(x)$ at the half-integers

$$
h(k)=\varphi(k / 2) \text {. }
$$

This is precisely the approach taken by Saito and Beyklin who considered a decomposition associated with the autocorrelation function of a Daubechies wavelet [6].

\subsection{Extensions for higher dimensional signals}

The extension of our approach in higher dimensions is based on (8) and does not use tensor products. Specifically, a single wavelet channel is computed at each step by subtraction of two successive lowpass components. The lowpass filtering is implemented by successive 1D filtering along the various dimensions of the data. This leads to a natural interpretation of the wavelet channels as pseudo-Laplacians (assuming that the filter $h$ is symmetrical) (cf. Fig 4). In this representation, contours are characterized by zero-crossings. This distinguishes our approach from the 2D dyadic wavelet transform of Mallat et al. in which the wavelet components provide the $x$ and $y$ components of the gradient [3].

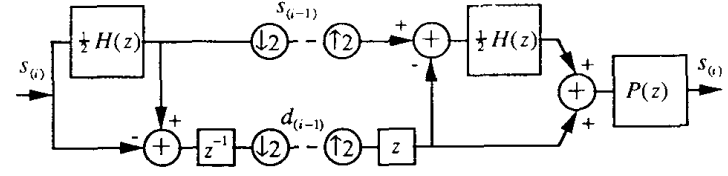

Fig. 3. Perfect reconstruction filter bank for a wavelet transform based on an interpolation filter.

\subsection{The sub-sampled representation}

Although the focus of this paper is on overcomplete decompositions, it is usually also possible to reconstruct the signal $s(k)$ from a sub-sampled representation. In fact, this is equivalent to considering the discrete wavelet transform with analysis filters $h$ and $g$. Such a wavelet transform can be implemented through the critically sampled perfect reconstruction filter bank shown in Fig. 3. The first part of the synthesis procedure uses the same filters as the analysis section. The post-filter $P(z)$ corrects for the fact that $H(z)$ does not satisfy the standard quadrature mirror filter condition required for an orthogonal wavelet transform. The explicit form of $P(z)$ is found by solving the perfect reconstruction equations, which yields

$$
P(z)=\frac{8}{H(z)^{2}+H(-z)^{2}} .
$$

Obviously, the system is reversible only if the filter $P(z)$ is stable; i.e., it has no poles on the unit circle. Note that the impulse response of this post-filter is usually infinite. In most cases, however, it can be implemented quite efficiently using a recursive algorithm (c.f., for example, [10]).

\section{EXAMPLES}

A "good" filter prototype $h$ should have a sufficient number of zeros at $z=-1$ to guarantee a certain degree of regularity of the underlying scaling function, and should also provide a good approximation of an ideal filter (subband decomposition). One possibility is to select an FIR filter; for instance, the autocorrelation of a Daubechies filter [6].

Instead, we chose to investigate the use of spline filters which have precisely the right properties. These filters are IIR but can still be implemented efficiently using the recursivealgorithm described in [11]. The transfer function of the polynomial spline interpolator of order $2 p+1$ is given by

$$
H_{2}^{2 p+1}(z)=\frac{B_{1}^{2 p+1}(z)\left(z+2+z^{-1}\right)^{p+1}}{B_{1}^{2 p+1}\left(z^{2}\right) \cdot 2^{2 p+1}}
$$

where $B_{1}^{n}(z)$ is the (FIR) transfer function of a B-spline of ordern. The corresponding scaling function is the cardinal (or fundamental) spline of degree $2 p+1$. Moreover, the spline 


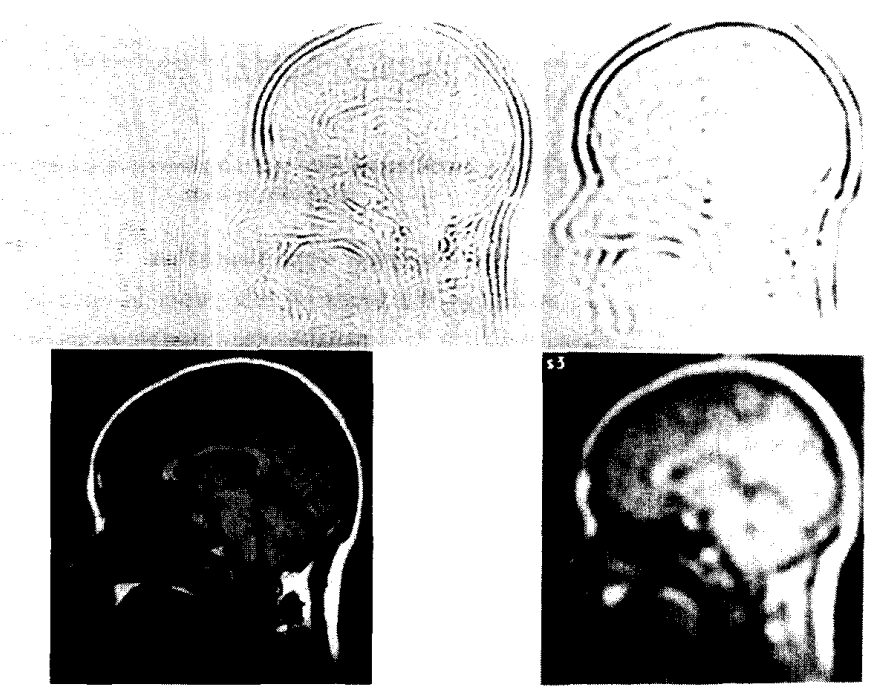

Fig. 4. Example of a subband decomposition obtained using a quintic spline interpolator $(p=2)$.

interpolators converge to an ideal lowpass filter as $p$ goes to infinity [12].

An example of quintic spline subband decomposition of an MRI image is shown in Fig. 4. The frequency response of the corresponding filter bank is the one displayed in Fig. 1.

\section{CONCLUSION}

The main feature of the multiband decomposition algorithm described in this paper is that it is extremely simple to implement. A direct benefit of using interpolation filters is a reduction of computational complexity by approximately a factor of four when compared with other multiband systems with similar frequency characteristics. The saving results from the fact that every other filter coefficient is zero and that the highpass filter can be computed by simple subtraction. Another attractive feature is the possibility of obtaining non-tensor product decompositions of higher dimensional signals that are non-directional.

This decomposition may be potentially useful for a variety of image processing tasks; for instance, multi-scale edge detection based on zero-crossings, texture analysis, adaptive filtering, and zero-crossing representation of images. We will consider some of these applications in the future.

\section{References}

[1] O. Rioul and M. Vetterli, "Wavelets and signal processing", IEEE Signal Processing Magazine, vol. 8, pp. 11-38, October 1991.

[2] M. Vetterli and C. Herley, "Wavelets and filter banks: theory and design", IEEE Trans. Signal Processing, vol. 40, pp. 2207-2232, September 1992.

[3] S. Mallat and W.L. Hwang, "Singularity detection and processing with wavelets", IEEE Trans. Information Theory, vol. 38 , pp. 617 643, March 1992.

[4] M.J. Shensa, "The discrete wavelet transform : wedding the a trous and Mallat algorithms", IEEE Trans. Signal Processing, vol. 40, pp. 2464-2482, October 1992.

[5] D. Marr and E. Hildreth, "Theory of edge detection", Proc. Roy. Soc. London, vol. B 207, pp. 187-217, 1980.

[6] N. Saito and G. Beyklin, "Multiresolution representations using the auto-correlation functions of compactly supported wavelets", in Proc. Int. Conf. Acoustics, Speech, and Signal Processing, San Franscisco, CA, March 23-26, 1992, pp. 381-384.

[7] O. Rioul, "Simple regularity criteria for subdivision schemes", SIAM J. Math. Anal, to appear.

[8] I. Daubechies, Ten lectures on wavelets. Philadelphia, PA: Society for Industrial and Applied Mathematics, 1992.

[9] S.G. Mallat, "A theory of multiresolution signal decomposition: the wavelet representation", IEEE Trans. Pattern Anal. Machine Intell., vol. PAMI-11, pp. 674-693, 1989.

[10] M. Unser, "An improved least squares Laplacian pyramid for image compression", Signal Processing, vol. 27, pp. 187-203, May 1992.

[11] M. Unser, A. Aldroubi and M. Eden, "Fast B-spline transforms for continuous image representation and interpolation", IEEE Trans. Pattern Anal. Machine Intell., vol. 13, pp. 277-285, March 1991.

[12] A. Aldroubi, M. Unser and M. Eden, "Cardinal spline filters : stability and convergence to the ideal sinc interpolator", Signal Processing, vol. 28, pp. 127-138, August 1992. 